\title{
Spacecraft drag modelling
}

DOI:

10.1016/j.paerosci.2013.09.001

\section{Document Version}

Accepted author manuscript

Link to publication record in Manchester Research Explorer

\section{Citation for published version (APA):}

Mostaza Prieto, D., Graziano, B. P., \& Roberts, P. C. E. (2014). Spacecraft drag modelling. Progress in Aerospace Sciences, 64, 56-65. https://doi.org/10.1016/j.paerosci.2013.09.001

\section{Published in:}

Progress in Aerospace Sciences

\section{Citing this paper}

Please note that where the full-text provided on Manchester Research Explorer is the Author Accepted Manuscript or Proof version this may differ from the final Published version. If citing, it is advised that you check and use the publisher's definitive version.

\section{General rights}

Copyright and moral rights for the publications made accessible in the Research Explorer are retained by the authors and/or other copyright owners and it is a condition of accessing publications that users recognise and abide by the legal requirements associated with these rights.

\section{Takedown policy}

If you believe that this document breaches copyright please refer to the University of Manchester's Takedown Procedures [http://man.ac.uk/04Y6Bo] or contact uml.scholarlycommunications@manchester.ac.uk providing relevant details, so we can investigate your claim.

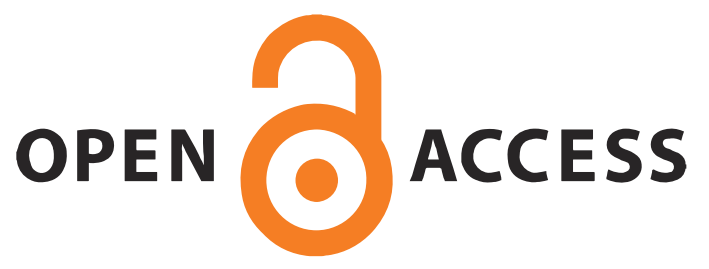




\title{
Spacecraft Drag Modelling
}

\author{
David Mostaza Prieto; Benjamin P. Graziano; Peter C. E. Roberts \\ Space Research Centre, Cranfield University
}

\begin{abstract}
This paper reviews currently available methods to calculate drag coefficients of spacecraft traveling in low Earth orbits (LEO). Aerodynamic analysis of satellites is necessary to predict the drag force perturbation to their orbital trajectory, which for LEO orbits is the second in magnitude after the gravitational disturbance due to the Earth's oblateness. Historically, accurate determination of the spacecraft drag coefficient $\left(C_{D}\right)$ was rarely required. This fact was justified by the low fidelity of upper atmospheric models together with the lack of experimental validation of the theory. Therefore, the calculation effort was a priori not justified. However, advances on the field, such as new atmospheric models of improved precision, have allowed for a better characterization of the drag force. They have also addressed the importance of using physically consistent drag coefficients when performing aerodynamic calculations to improve analysis and validate theories. We review the most common approaches to predict these coefficients.
\end{abstract}

Keywords: Spacecraft drag; Free Molecular Flow; Rarefied Aerodynamics

\section{Introduction}

The study of the upper atmosphere is a key issue on the determination and prediction of spacecraft trajectories. The drag force resulting from the interchange of momentum between the upper atmosphere and spacecraft reduces the orbital energy. The result is a change of the eccentricity towards a more circular orbit, together with a reduction of the semi major axis until the eventual re-entry of the spacecraft.

Preprint submitted to Progress in Aerospace Sciences

September 7, 2016 
Although it is known that in LEO orbits the drag force is the biggest source of perturbation after the $J_{2}$ coefficient of the gravitational potential function of the Earth, an accurate prediction and determination of its magnitude is still challenging. A common approach to calculate the drag acceleration experienced by a body is:

$$
a_{\text {drag }}=\frac{1}{2} \rho V^{2} C_{D} \frac{S}{m}
$$

Where $\rho$ represents the atmospheric density, $V$ is the relative velocity of the vehicle with respect to the atmosphere, $C_{D}$ is the drag coefficient, $S$ is the reference surface area and $m$ the mass of the body. A good estimation of $a_{d r a g}$ is often difficult to obtain. This is due to the large uncertainties associated with three of the above parameters: density, drag coefficient and velocity of the body with respect to the atmosphere:

- Neutral density of the upper atmosphere, $\rho$, is the major source of error in the determination of the drag force. The atmosphere is a moving mass of air, and its density varies with time, altitude and geographical location. The main driver of these changes at satellite altitudes is the varying extreme ultraviolet (EUV) radiation from the sun since temperature and hence density are affected by the amount of energy absorbed in the upper atmosphere. The result is a long term trend related to the 11 years solar cycle together with more transient and localised effects due to diurnal, seasonal, longitudinal, and latitudinal variations in atmospheric conditions. Also geomagnetic fluctuations caused by solar storms, which take place more frequently during periods of peak solar activity, have been shown to increase thermospheric density several factors [14]. Historically few data have been available and, as a consequence, upper atmosphere neutral density models are not precise. Its current accuracy is lies between $10 \%$ and $15 \%$ in mean activity conditions [38], however it can go up to $100 \%$ for short term and local variations [1]. The early upper atmospheric models 
were derived from orbit tracking of different satellites, with the density computed assuming a constant $C_{D}$ and neglecting winds [22]. For a good review of available atmospheric models and atmospheric modelling issues refer to $[11,16,17,22,38]$.

- Relative velocity, $V$. A common assumption to calculate relative velocity is to consider that the atmosphere rotates with the Earth. The presence of wind adds uncertainty to the problem. In-track winds can either increase or decrease the drag force depending on the blowing direction. It has been demonstrated that strong winds of several hundred meters per second can take place in the upper atmosphere (specially at high latitudes) and very little prediction capability is available to date $[12,20]$.

- Drag coefficient, $C_{D}$. It has been a common practice to assume a constant $C_{D}$ equal to 2.2 for low earth orbit flying satellites. Due to the lack of precision of existing atmospheric density models, any modelling effort to refine the drag coefficient was normally considered of little advantage, since it does not compensate for the imprecise density model. Nowadays, it is widely accepted that the drag coefficient is not constant and can present very different values depending on the spacecraft shape and the atmospheric temperature and composition at the flying altitude. Note that atmospheric density models obtained from satellite observations, directly incorporate any error on the drag coefficient as density biases.

Despite the described difficulties, in recent times there has been an increased interest in spacecraft drag modelling. We have entered the 21st century inside a Golden Age of Satellite Drag [17] mainly due to new dedicated space missions together with more and more available data and a better understanding of the solar phenomena. Many research groups have become interested in the topic in the last few years, and even more interest is foreseen in the future. Any improvement on the knowledge of this disturbance will directly impact the accuracy of mission analysis and orbital predictions. This will result, for instance, 
in better orbit determination and tracking of space objects (allowing for example optimum and precise collision avoidance manoeuvres), in more accurate determination of re-entry windows and debris footprints or in finer optimisation of fuel budgets. A comprehensive aerodynamic characterization of the spacecraft is also needed to study new mission concepts in which the aerodynamic force plays an active roll, such as drag compensation, sub-orbital flights, drag optimization, or trajectory and attitude control by means of aerodynamic forces and torques.

This paper is focused on the numerical and analytical calculation of $C_{D}$ (and associated aerodynamic coefficients) for bodies traveling in low Earth orbit.

\section{Characterising the environment}

The neutral atmospheric region of interest in the study of satellite aerodynamics is the thermosphere, a high-altitude layer that exist above around 85 $\mathrm{km}$. The thermosphere absorbs the EUV energy from the Sun resulting in a temperature profile increasing rapidly with altitude at its lower part (below around $200 \mathrm{~km}$ ). In its upper region, the temperature increase reaches a limiting value (exospheric temperature) and remains constant with altitude due to the the infrequency of intermolecular collisions that occur as a result of the very low density. The temperature and neutral density vary with the amount of energy received by the the thermosphere. The main energy sources affecting its structure are the the solar flux and the geomagnetic activity.

The biggest variation in solar flux is due to the 11 years solar cycle, temperature and neutral density tend to follow the same trend. Variations in mean density profiles for low, mean, and high levels of solar and geomagnetic activities, as defined by [1], are illustrated in Fig. 1. Density variations increase with altitude; notice that for an altitude of $600 \mathrm{~km}$, the atmospheric density varies by up to almost two orders of magnitude. This density increase during high solar activity periods is the dominant factor affecting spacecraft aerodynamic performance [41]. One well-known consequence of this variation is that during 


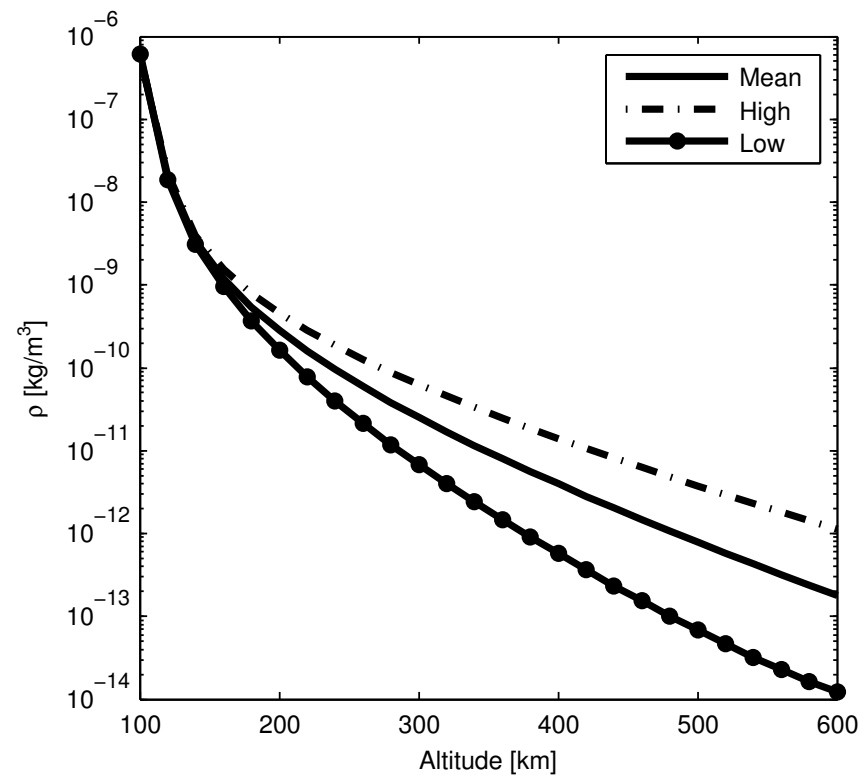

Figure 1: Variation of the mean atmospheric density with altitude for low, moderate and high solar and geomagnetic activities as defined by JB2006 model. Reproduced from [1]

these periods, satellites in low Earth orbit deorbit more quickly and have their operational lifetimes reduced as a result.

Two indices are generally used to measure the solar radiation and geomagnetic activity levels:

- F10.7 index for solar flux: It is a measure of the solar flux emitted at a wavelength of $10.7 \mathrm{~cm}$. Since the EUV radiation is absorbed in the thermosphere it is difficult to obtain a measurement of the solar flux at these wavelengths using instruments at the Earth's surface. It has been found that the F10.7 index presents a good correlation with solar activity [36]. Currently it is used as a proxy for EUV radiation in atmospheric models.

- Ap index for geomagnetic activity: This index is a measure of the general level of geomagnetic activity on the Earth for a given day. It is obtained from measurements of the magnetic field variations made at different lo- 


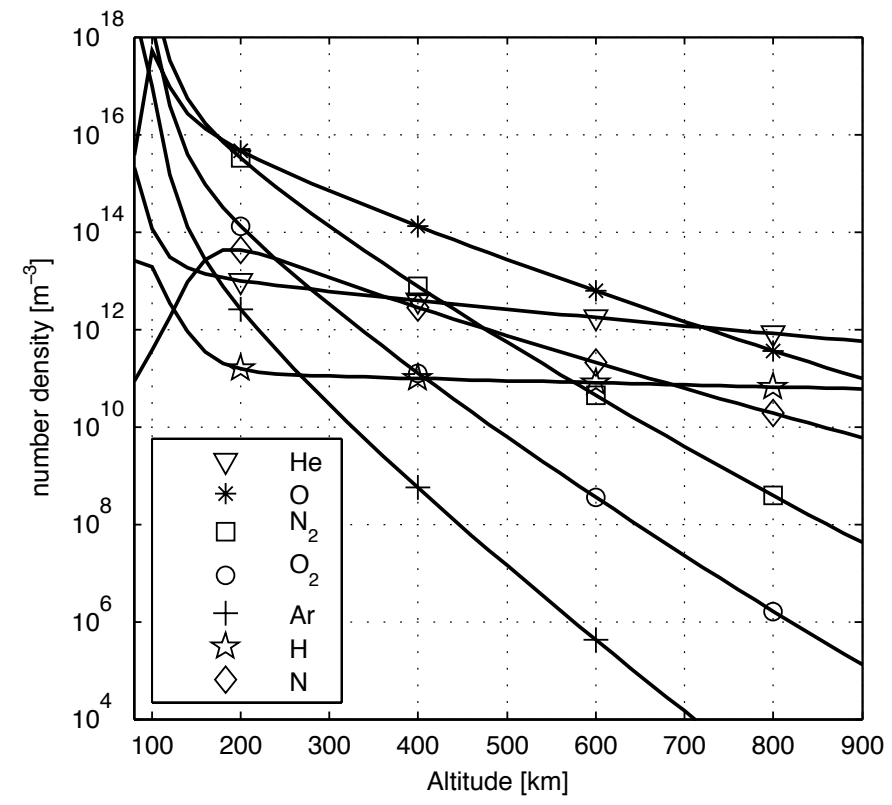

Figure 2: Variation of the Earth's atmospheric composition with altitude as defined by NRLMSISE00 model. Reproduced from [1]

cations. Geomagnetic storms are characterized by a sudden increase of this index.

At the considered altitudes, the composition of the atmospheric gas mixture varies with altitude, as illustrated in Fig. 2. Lighter molecules are faster, due to acquiring the same energy as heavier molecules on average from collisions. The faster molecules have a different scale height and are also lost to space more easily. In addition to these variations, atmospheric composition with altitude will fluctuate as the atmosphere expands and contracts under the influence of the solar cycle or geomagnetic activity.

Several models to predict atmospheric characteristics at spacecraft altitudes have been published since the 1960s. They provide values for neutral density and temperature as a function of the spacecraft position, solar radiation and geomagnetic activity. Two of the most modern models are briefly presented 
here: the NRLMSISE00 and the JB2006.

- NRLMSISE00 [28]. This model provides temperature and gas species number densities (for $\mathrm{He}, \mathrm{O}, \mathrm{N}_{2}, \mathrm{O}_{2}, \mathrm{Ar}, \mathrm{H}$ and $\mathrm{N}$ ) covering all the range from sea level up to the exosphere. Inputs to the model are altitude, latitude, longitude and the two indices $F 10.7$ and $A p$. A component named "anomalous oxygen" is introduced in the model for drag estimation. It accounts for the contribution of non thermospheric species to the drag at high altitudes, such as $\mathrm{O}^{+}$and hot oxygen (energetic oxygen atoms resulting from photochemical processes in the upper atmosphere [42]).

- JB2006 [6]. This models provides neutral density and temperature from $120 \mathrm{~km}$ to the exosphere. Input parameters are $F 10.7$ and $A p$, however, it also incorporates new solar indices $\left(S_{10}\right.$ and $\left.M g_{10}\right)$ to obtain better density variation correlations with UV radiation together with a model of the semi annual density variation [4]. A further improvement in the modeling and results is the JB2008 version [5].

According to the ECSS standard on Space environment [1], the NRLMSISE00 model shall be used for calculating neutral temperature, detailed composition and total density below $120 \mathrm{~km}$, whereas the JB2006 model shall be used for calculating the total density above $120 \mathrm{~km}$. It is worth mentioning that atmospheric models up to date only predict slow time variations and large scales.

\subsection{Free Molecular Flow}

The highly rarefied atmosphere of low Earth orbit requires a different approach to aerodynamics than that employed in the continuum regime that exists at aircraft flight altitudes. Due to the low density, the flow regime of low Earth orbit spaceflight is commonly described as free molecular. This means that the mean-free path (the mean distance between consecutive collisions, which is illustrated in Fig. 3) is many times greater than the characteristic dimension of a body immersed in the flow. Therefore, collisions between molecules 


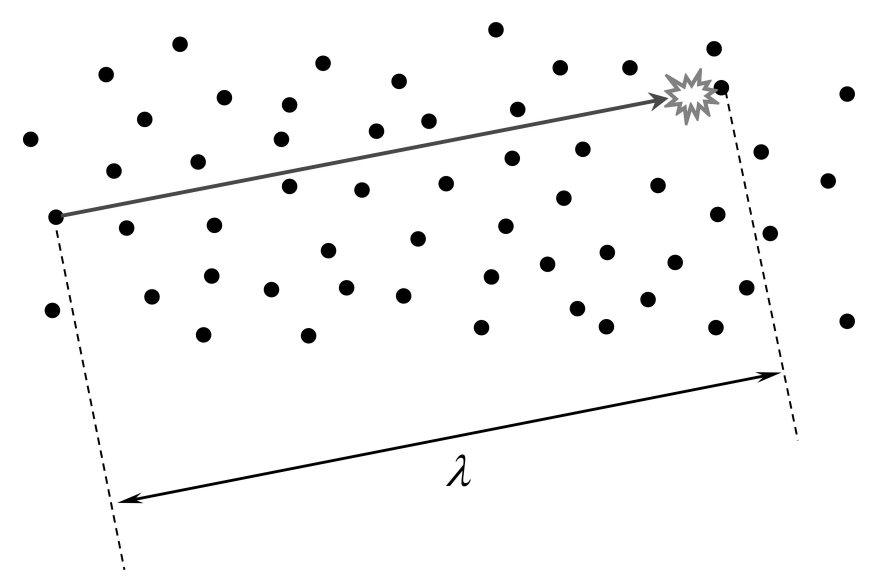

Figure 3: Molecular Mean Free Path $(\lambda)$

are extremely rare in the flow field around the body, such that the flow can be assumed collisionless and it cannot be considered as a continuum medium anymore. Instead it is particulate in nature.

To quantify the validity of the collisionless assumption a non-dimensional parameter known as Knudsen number is used.

$$
K n=\frac{\lambda}{l_{\text {ref }}}
$$

The term $l_{\text {ref }}$ is the characteristic dimension of the spacecraft and $\lambda$ is the mean free path. A high $K n$ indicates that the flow is particulate in nature (i.e. free molecular) and that the collisionless Boltzmann equation should be employed, a low $K n$ indicates that the flow is continuum in nature and should be analysed using the Navier-Stokes equations. It is generally assumed in most rarefied gas dynamics literature that the free molecular flow assumption is valid for $K n \geq 10$ [25]. Fig. 4 shows the Knudsen number variation with altitude and Fig. 5 illustrates how Knudsen number can be used to describe rarefied flow regimes.

Another important parameter in free molecular flow is the molecular speed 


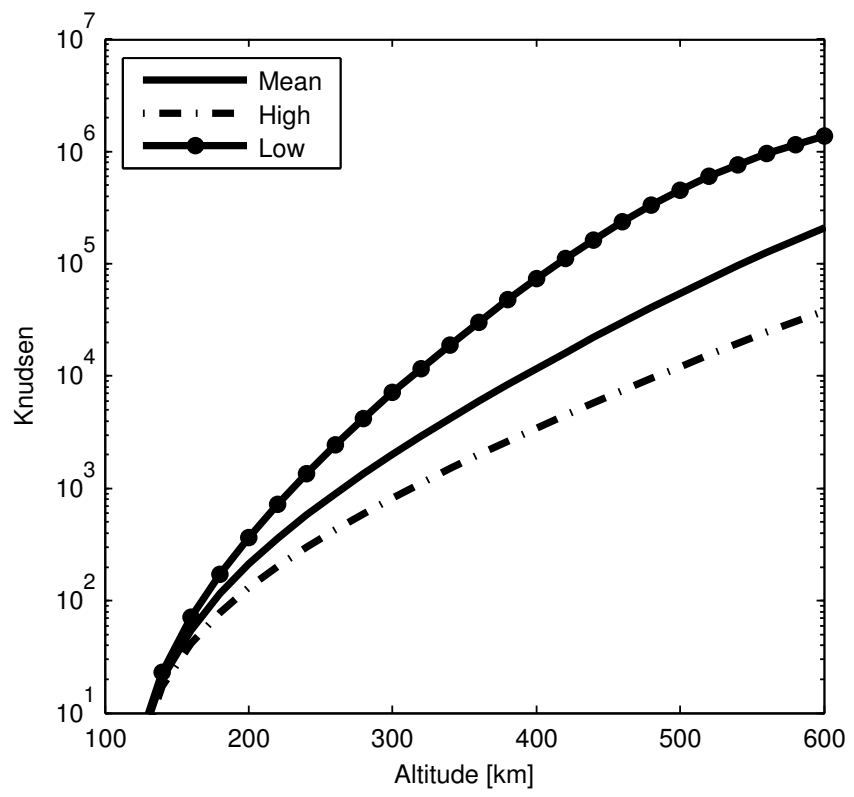

Figure 4: Knudsen number variation with altitude for low, moderate and high solar and geomagnetic activities using NRLMSISE-00, and a characteristic dimension of $1 \mathrm{~m}$

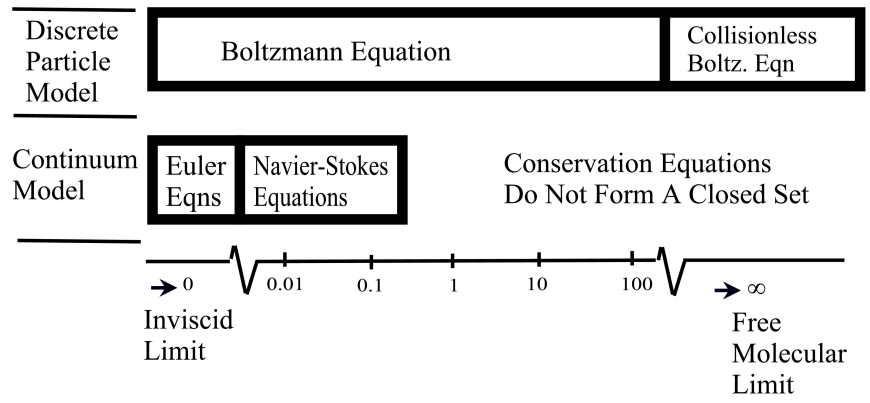

Figure 5: Classification of flow regimes using Knudsen number. Reproduced from [2] 
ratio:

$$
s=\frac{V}{V_{a}}
$$

Where $V_{a}$ is the thermal speed, which defines temperature as a measure of the most probable molecular speed of a gas (moving in a reference frame with the gas at its bulk velocity, $V$ ). It can be shown, using the kinetic theory of gases and the Boltzmann equation, that for an equilibrium gas, with a Maxwellian distribution of velocities, the most probable molecular speed of the gas is given by Eq. (4) [2] .

$$
V_{a}=\sqrt{2 R_{s p} T}
$$

The term $R_{s p}$ is the specific gas constant in $\mathrm{J} \mathrm{kg}^{-1} \mathrm{~K}^{-1}$ and $T$ is the temperature in $\mathrm{K}$. The molecular speed ratio $s$ provides an indication of the extent to which the flow behaves like a collimated beam of molecules (hyperthermal flow) where the bulk velocity of the gas is many times greater than the thermal velocity of the gas, (Fig. 6 top), or a chaotic drifting Maxwellian flow (hypothermal flow), where the high random thermal motion of the atmospheric gas constituents means that the free stream gas flow cannot be treated as collimated beam of molecules anymore (Fig. 6 bottom).

At higher altitudes, the thermal velocity of the atmosphere increases with temperature towards the thermopause, and then continues to increase with altitude through the exosphere as mean molar mass decreases. In this scenario, thermal speed increases such that the molecular speed ratio decreases $(s<<\infty)$ and the flow may be described as hypothermal.

For practical implementations, it is generally assumed in most texts that the hyperthermal flow assumption is valid for $s>5$, such that the molecular Mach angle is less than $11 \mathrm{deg}$ (see, for example, [8]).

In hypothermal flow $(s<5)$, all surfaces may be impinged by molecules due 


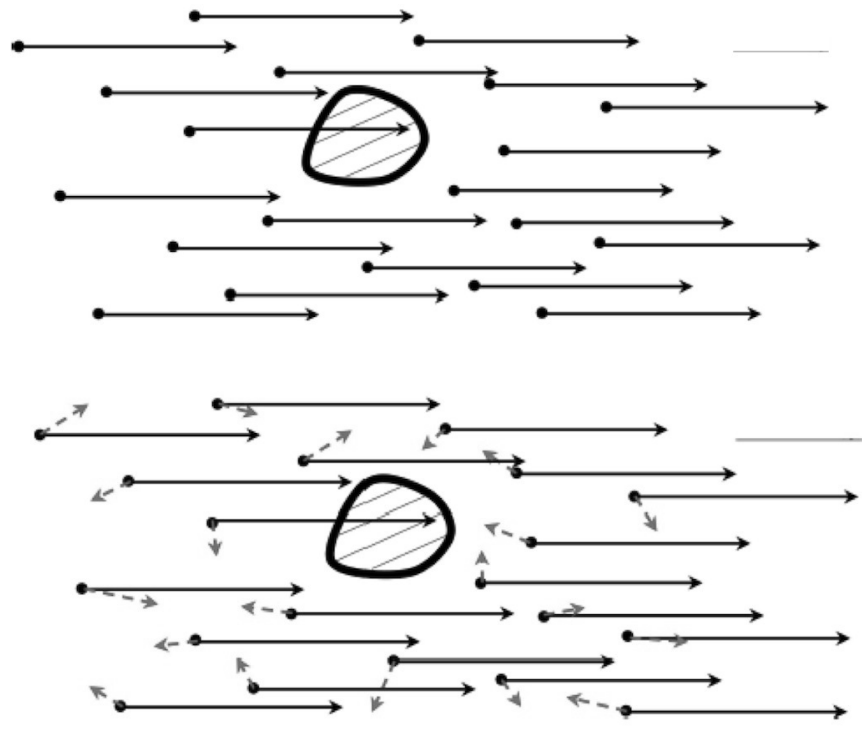

Figure 6: Hyperthermal $(s \rightarrow \infty)$ and hypothermal $(s<<\infty)$ flows

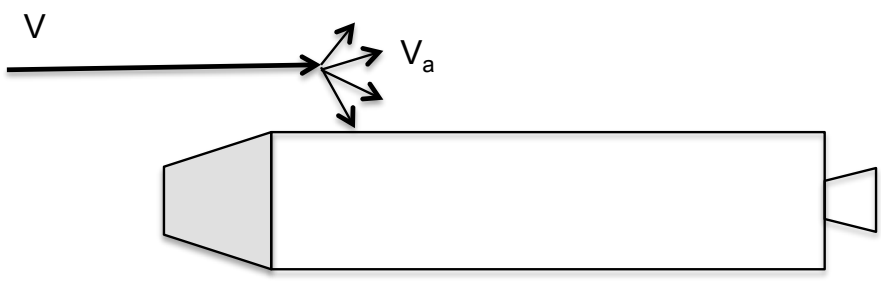

Figure 7: Impact on parallel surface due to random thermal motion of the flow molecules

to their random thermal motion regardless of whether they are forward facing or not. In practice, most molecules arrive at forward facing surfaces (shown in dark in Fig. 7 ), and a smaller amount of momentum is imparted to aft facing surfaces and surfaces parallel to the flow due to the random thermal motion of molecules. This is particularly important for slender bodies. 


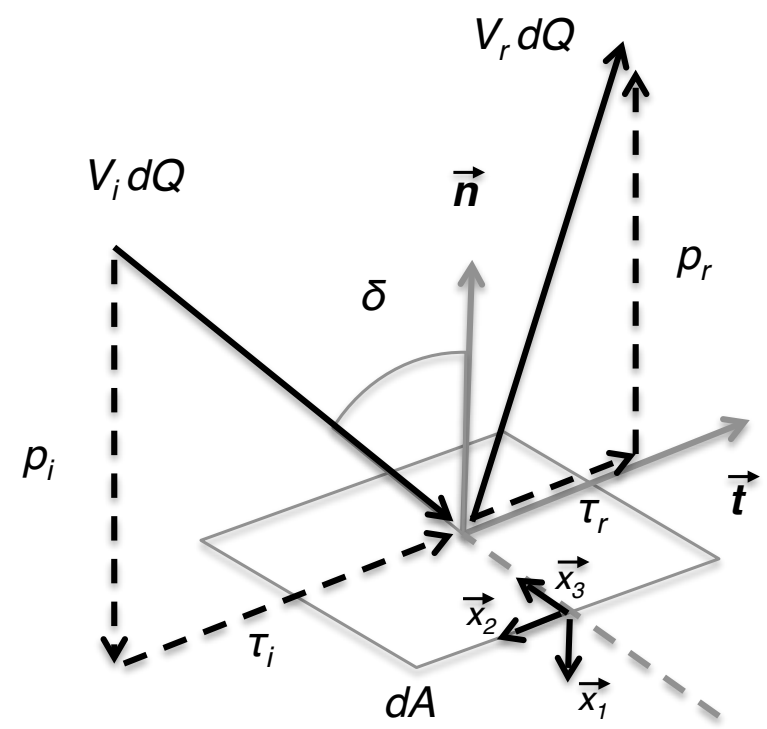

Figure 8: Incident and reflected fluxes on a convex element of area

\section{Interaction between the body and the flow}

As it was mentioned previously, in the free molecular flow regime, collisions between molecules are extremely rare, even between incident and reflected particles. Consequently, reflected particles will have a negligible influence on the incident flow. The gas particles impact the surface transferring energy and momentum to the body. Therefore, free molecular flow is dominated by the nature of the Gas-Surface Interactions (GSIs) that takes place.

Assuming gas-surface interaction only, the force on the surface is equal to the rate of change of momentum of the gas (incident-reflected). For a convex body the force per unit area is related to the incident and reflected momentum fluxes as follows (Fig. 8):

$$
\frac{d \vec{f}}{d A}=\left(p_{i}+p_{r}\right) \vec{n}+\left(\tau_{i}-\tau_{r}\right) \vec{t}
$$

Normal momentum flux is represented by $p$ and tangential momentum flux by $\tau$. 
The subscripts $i$ and $r$ refer to incident and reflected flux respectively. Normal and tangential incident momentum fluxes $\left(p_{i}, \tau_{i}\right)$ depend on the incident velocity and the mass flux $(d Q)$. The reflected momentum flux is $-p_{r} \vec{n}+\tau_{r} \vec{t}$.

The mathematical description of the incident fluxes, even in hypothermal flow, is a well-known and solved problem $[30,33,2]$. In the case of hyperthermal flow assumption (i.e. no thermal motion of the molecules) $p_{i}=V \cos \delta d Q$ and $\tau_{i}=V \sin \delta d Q$, where $d Q=\rho V \cos \delta$. The most common solution for the hypothermal case considers a Maxwellian flow, where the thermal velocities of the molecules of the gas are specified by the Maxwellian velocity distribution function. Representing the thermal velocity of the molecules in the cartesian system defined in Fig. 8 by $\vec{u}=\left(u_{1}, u_{2}, u_{3}\right)$, the incident momentum fluxes are given by:

$$
\begin{gathered}
p_{i}=\int_{0}^{\infty} \int_{-\infty}^{\infty} \int_{-\infty}^{\infty} m u_{1}^{2} F(\vec{u}) d u_{1} d u_{2} d u_{3} \\
\tau_{i}=\int_{0}^{\infty} \int_{-\infty}^{\infty} \int_{-\infty}^{\infty} m u_{1} u_{2} F(\vec{u}) d u_{1} d u_{2} d u_{3}
\end{gathered}
$$

Where $m$ is the mass of a single gas molecule and $F(\vec{u})$ is a Maxwellian distribution function given by [30]:

$$
F(\vec{u})=n(2 \pi R T)^{3 / 2} \exp \left\{-\left[\left(u_{1}-V \cos \delta\right)^{2}+\left(u_{2}+V \sin \delta\right)^{2}+u_{3}\right] / 2 R T\right\}
$$

Integrating Eqs. (6) and (7) the incident fluxes are:

$$
\begin{gathered}
p_{i}=\frac{1}{2} \frac{\rho V_{i}^{2}}{s^{2}} \Gamma_{1}(s \cos \delta) \\
\tau_{i}=\frac{1}{2} \frac{\rho V_{i}^{2}}{s} \sin \delta \Gamma_{2}(s \cos \delta)
\end{gathered}
$$

Where:

$$
\Gamma_{1}(x)=\frac{1}{\sqrt{\pi}}\left[x \exp \left(-x^{2}\right)+\frac{\sqrt{\pi}}{2}\left(1+2 x^{2}\right)(1+\operatorname{erf} x)\right]
$$




$$
\begin{gathered}
\Gamma_{2}(x)=\frac{1}{\sqrt{\pi}}\left[\exp \left(-x^{2}\right)+\sqrt{\pi} x(1+\operatorname{erf} x)\right] \\
\operatorname{erf} x=\frac{2}{\sqrt{\pi}} \int_{0}^{x} e^{-t^{2}} d t
\end{gathered}
$$

Having solved the incident fluxes, the key point of the problem is to determine the reflected fluxes. This is a very difficult problem. The gas-surface interaction that takes place is driven by extremely complex phenomena that are not yet well understood. Therefore, the different analyses methods available for calculating aerodynamic quantities in rarefied flow rely upon simplified mathematical models of gas-surface interactions (GSIMs).

In simple terms, the challenge of developing a successful GSIM is to model the exchange of energy and momentum between molecules and surfaces due to impact and reemission. In practice, this means that in rarefied flow at satellite speeds a number of factors must be considered, including:

- Gas properties (chemical composition, molar mass, ratio of specific heats, number of degrees of freedom, density, temperature, bulk speed).

- Surface properties (chemical composition, roughness, cleanliness, and temperature).

- Angle of incidence between the flow vector and the surface.

Several GSIMs have been published in the literature with different levels of complexity, some reviews can be found in [34, 27]. We present hereafter the most simple and widely used: Maxwell, Schamberg and Schaaf and Chambre. Notice that the first two models describe the scattering geometry of the reflected molecules, while the Schaaf and Chambre model does not provide (or need) such characterization.

\subsection{Maxwell Model}

The Maxwellian model is the most popular gas-surface interaction model for analysing spacecraft in the free molecular flow regime [40]. It is the reduction 


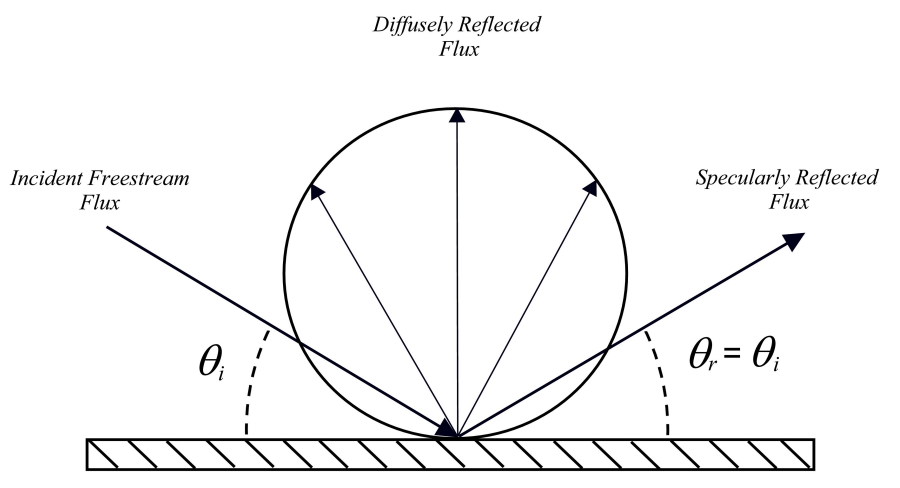

Figure 9: Specular and diffuse reflected fluxes

of the highly complex gas-surface interaction to apparently simple parameters that underpins the popularity of the Maxwellian model in the fields of both theoretical analysis and experiment. It is presented in various forms in [19, 29, 18] and elsewhere. In 1879 Maxwell developed a GSIM in which he postulated that a portion $f$ of every unit area of surface reflects molecules diffusely, and a portion $(1-f)$ specularly, as illustrated in Fig. 9.

In specular reemission, the angle of the reemitted particle equals the angle of incidence, whereas in diffuse reemission the molecule is reflected with a random distribution of speed and a direction which follows the Knudsen cosine law distribution (the number of molecules emitted between the angles $\theta$ and $\theta+\delta \theta$ from the normal to the surface is proportional to $\cos \theta \delta \theta$ ).

According to Maxwell whereas the specular portion perfectly reflects the molecules, in the case of diffuse reflection the surface absorbs all the incident molecules allowing them to evaporate afterwards with velocities corresponding to a gas at the temperature of the surface. In other words, the diffuse reflected molecules are completely accommodated to the surface temperature before being reemitted. The average velocity of the diffuse reflected molecules is offered in Eq. (14), calculated by means of the Maxwell-Boltzman speed distribution function $[34]$. 


$$
V_{w}=\sqrt{\pi R T_{w} / 2}
$$

Where $R$ is the gas constant, and $T_{w}$ the surface temperature.

A further step in the model is the concept of incomplete thermal accommodation, in which the reflected molecule has a different average velocity with respect to the accommodated case [39]. The extent of energy transfer to a surface by an incident molecular flux can be described by the energy accommodation coefficient $\alpha$, given by Eq. (15).

$$
\alpha=\frac{E_{i}-E_{r}}{E_{i}-E_{w}}
$$

The terms $E_{i}$ and $E_{r}$ are the incident and reflected energy fluxes respectively. The term $E_{w}$ is the energy flux that would be carried away if all the molecules were reemitted diffusely in thermal equilibrium with the surface, such that they have the same temperature as the surface $T_{w}$. Complete thermal accommodation implies $\alpha=1$, whereas and no energy exchange implies $\alpha=0$. Diffuse reflection without complete thermal accommodation is sometimes referred as quasi-diffuse and specular reflection with $\alpha>0$ as quasi-specular. Notice that the magnitude of the average reflected velocity depends on the amount of energy transferred and thus on the value of the accommodation coefficient.

\subsection{Schamberg Model}

Schamberg [32] proposes a more general model of gas-surface interaction. After striking the surface the molecules are reemitted with the scattering pattern of a half cone, obeying the reflected angles the Knudsen cosine distribution (Fig. 10), $V_{i}$ and $\theta_{i}$ are the incident velocity and angle of incidence and $V_{r}$ and $\theta_{r}$ are the reemitted velocity and the angle of the mean reemitted beam. The shape of

the beam is characterized by the function $\Phi\left(\phi_{0}\right)$ given by Eq. (16) for a conical beam. Incident and reflected angles are related by the parameter $\nu$ (Eq. (17)). 


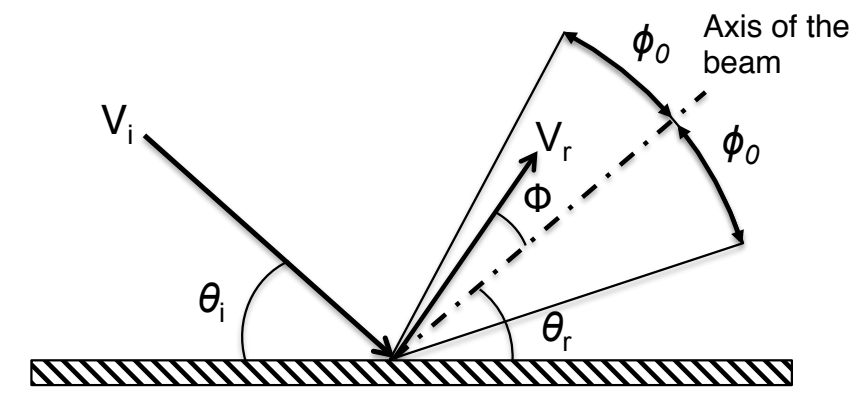

Figure 10: Schamberg's GSIM

$$
\begin{gathered}
\Phi\left(\phi_{0}\right)=\frac{1-\left(2 \phi_{0} / \pi\right)^{2}}{1-4\left(2 \phi_{0} / \pi\right)^{2}} \cdot \frac{\frac{1}{2} \sin 2 \phi_{0}-\left(2 \phi_{0} / \pi\right)}{\sin \phi_{0}-\left(2 \phi_{0} / \pi\right)} \\
\cos \theta_{r}=\left(\cos \theta_{i}\right)^{\nu}
\end{gathered}
$$

Specular reflection is obtained when $\nu=1$ and $\phi_{0}=0(\Phi(0)=0)$, for diffuse reflection $\nu=\infty$ and $\phi_{0}=\pi / 2(\Phi(\pi / 2)=3 / 2)$. Schamberg's model assumes uniform reemission speed for all directions.

\subsection{Schaaf and Chambre model}

A slightly different approach to the problem was proposed by Schaaf and Chambre [30]. They introduced two coefficients to describe the extent of normal and tangential momentum transfer to the surface. Their phenomenological coefficients, provided by Eqs. (18 - 19), separate the effects of incomplete normal and tangential momentum transfer. These coefficients allow for a characterisation of the force on the surface, which can be obtained by experiments without any assumption on the scattering of the reflected molecules.

$$
\begin{gathered}
\sigma_{N}=\frac{p_{i}-p_{r}}{p_{i}-p_{w}} \\
\sigma_{T}=\frac{\tau_{i}-\tau_{r}}{\tau_{i}-\tau_{w}}=\frac{\tau_{i}-\tau_{r}}{\tau_{i}} ;\left(\tau_{w}=0\right)
\end{gathered}
$$


The term $\sigma_{N}$ is usually referred to as the normal momentum accommodation coefficient and $\sigma_{T}$ as the tangential momentum accommodation coefficient, $p_{w}$ is the accommodated normal momentum which only depends on surface temperature. By definition of diffuse reflection $\tau_{w}=0$, since the speed distribution is symmetrical around the surface normal. Consequently, for complete specular reflection with no energy exchange (elastic collision) $\sigma_{N}=\sigma_{T}=0$. Whereas, for complete diffuse reflection, in which the energy of the molecules completely accommodates to the surface $\sigma_{N}=\sigma_{T}=1$. Diffuse reflection only implies $\sigma_{T}=0$, while $\sigma_{N}$ remains unspecified.

\subsection{Angular Distribution and Accommodation Coefficient in Space Environ-} ment

In the space environment the angular distribution of reemitted molecules and the accommodation are mainly affected by the molar mass of the incident gas and a process known as adsorption. Adsorption describes the process by which satellite surfaces in low Earth orbit become covered by atmospheric molecules that are trapped close to the surface. Due to the surface contamination caused by adsorption more energy is lost to the surface by incident molecules on impact. Therefore, the effects of adsorption are a broader angular distribution of reemitted molecules, which is closer to the diffuse case, and a higher accommodation of the incident molecules to the surface [22]. Also, while for clean surfaces the accommodation coefficient is strongly dependent on surface material, laboratory experiments had shown that the accommodation coefficient for contaminated surfaces is almost constant regardless of the material [23].

Angular distribution and accommodation coefficient vary with altitude due to changes in atmospheric composition and, in the case of adsorption, changes in density (Fig. 11). Adsorption is more pronounced at some altitudes since some atmospheric constituents are more likely to be adsorbed than others. The

prime case of this is atomic oxygen [37], which is prevalent at altitudes between approximately 200 and $700 \mathrm{~km}$ in mean solar conditions. 


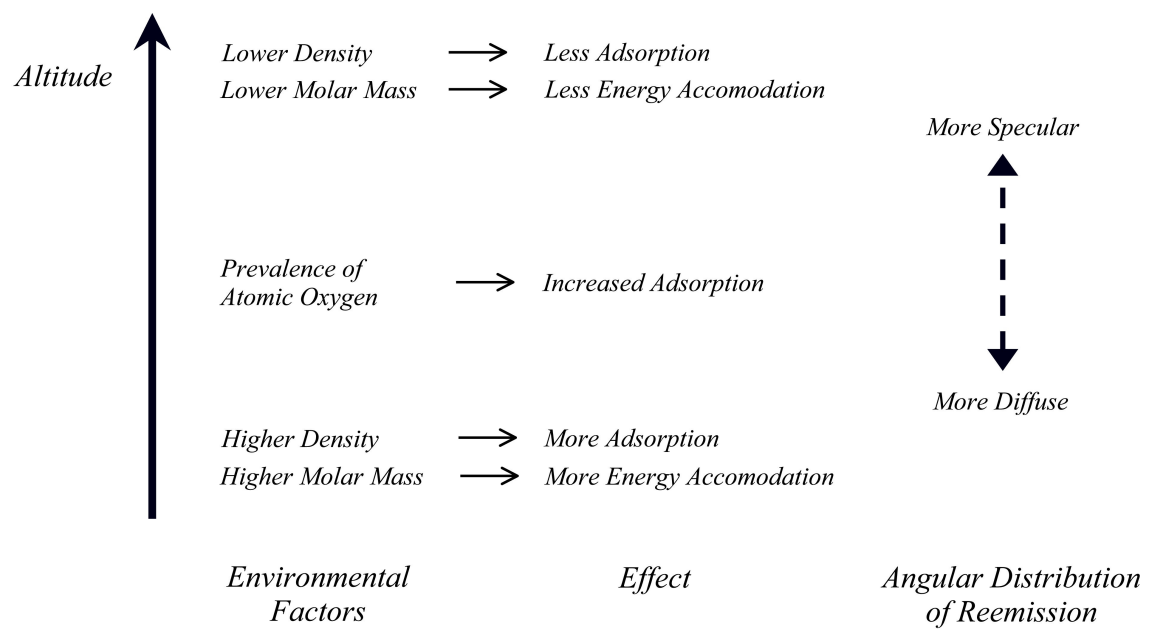

Figure 11: Schematic of the main environmental factors affecting accommodation coefficient and angular distribution in low earth orbit

Due to these factors, it is widely accepted that the satellite surfaces are contaminated in LEO orbits. Therefore, two common assumption when calculating drag coefficients for LEO orbit are:

- Angular distribution of the molecules is close to the diffuse case.

- Accommodation coefficient is close to one and independent of the surface material.

However, both assumptions become less valid as the altitude increases. Satellite surfaces are less contaminated and the uncertainty on both the distribution of reflected particles and the accommodation coefficient increases. An estimation of the uncertainty caused by the increasing fraction of specular reflection on the drag coefficient of an sphere as the altitude increases was calculated by Moe et al. [22] and reproduced in Fig. 12. 


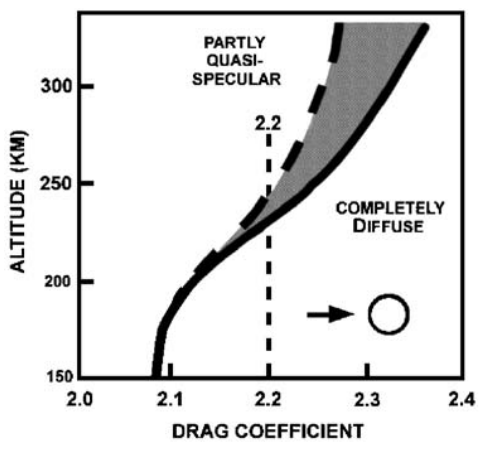

Figure 12: Uncertainties in drag coefficient caused by quasi-specular remission. Reproduced from $[22]$

\section{Solving the problem}

\subsection{Analytical Methods}

Two common approaches are presented here, Schamberg [32] and Schaaf and Chambre [30] . They represent two different ways of obtaining analytical expressions for the drag and lift coefficients of convex bodies in FMF . To some extent, almost all the derivations and applications of spacecraft aerodynamic modelling in the literature are based on one of these two models.

\subsubsection{Schamberg's derivation}

Schamberg [32] derives analytical expressions for the drag coefficient for different elementary shapes in hyperthermal flow based on his GSIM (presented in section 3.2). The resulting expressions are complex and depend upon the GSIM coefficients. The resulting reflected fluxes for a flat plate can be obtained with the help of Fig. 10:

$$
\begin{gathered}
p_{r}=\rho V_{i}^{2} \sin ^{2} \theta_{i} \Phi\left(\phi_{0}\right) \frac{V_{r}}{V_{i}} \frac{\sin \theta_{r}}{\sin \theta_{i}} \\
\tau_{r}=\rho V_{i}^{2} \sin \theta_{i} \cos \theta_{i} \Phi\left(\phi_{0}\right) \frac{V_{r}}{V_{i}} \frac{\sin \theta_{r}}{\sin \theta_{i}}
\end{gathered}
$$


Eq. (22) presents the general form of Schamberg's hyperthermal drag coefficient for all shapes based on projected area.

$$
C_{D}=2\left[1+\Phi\left(\phi_{0}\right) \frac{V_{r}}{V_{i}} f(\nu, \text { shape })\right]
$$

The parameters $\nu$ and $\Phi\left(\phi_{0}\right)$ are the same ones defined in section 3.2. The ratio $V_{r} / V_{i}$ (reflected and incident molecule velocities) can be related with the accommodation coefficient as follows [26] (notice the constant reflected velocity assumption):

$$
\alpha=\frac{E_{i}-E_{r}}{E_{i}-E_{w}}=\frac{\frac{1}{2} m V_{i}^{2}-\frac{1}{2} m V_{r}^{2}}{\frac{1}{2} m V_{i}^{2}-E_{w}}
$$

Assuming that the ratio $E_{w} /\left(1 / 2 m V_{i}^{2}\right)$ is numerically negligible we can relate the velocity ratio $(r)$ and the accommodation coefficient:

$$
r=\frac{V_{r}}{V_{i}}=\sqrt{1-\alpha}
$$

Generally, for practical applications, the definition of $\nu$ and $\phi_{0}$ is difficult and some further simplifications are needed. We can fix these values to their diffuse and specular extremes resulting in two different expressions for quasi-specular and quasi-diffuse reflections, since they will still depend on the accommodation coefficient. Shamberg's quasi-diffuse model based on the hyperthermal assumption has been very popular. Cook [8] used this model in what has become a key reference to calculate satellite drag coefficients. He calculates $r$ in a sightly different way:

$$
\begin{gathered}
r=\frac{V_{r}}{V_{i}}=\left(\frac{E_{r}}{E_{i}}\right)^{1 / 2}=\left\{1+\alpha\left(\frac{E_{w}}{E_{i}}-1\right)\right\}^{1 / 2} \\
\frac{E_{w}}{E_{i}}=\frac{T_{w}}{T_{k, i}}
\end{gathered}
$$

Where $T_{w}$ is the already defined wall temperature and $T_{k, i}=1 /(3 R) V_{i}^{2}$ is 


\begin{tabular}{clc}
\hline Shape & (based on projected area perpendicular to direction \\
of motion)
\end{tabular}

Figure 13: Schamberg's quasi-specular and quasi diffuse drag coefficients for simple geometries (based on projected area). Reproduced from [8]

the kinetic temperature. Fig. 13 offers drag coefficients for different shapes for quasi-diffuse and quasi-specular reflections under hyperthermal conditions. Notice that this model provides with a description of the angular distribution of the reflected particles. Schamberg's model is a very adaptive model capable of describing a wide range of different scattering patterns. However, besides the two extreme cases, diffuse and specular, it is not straightforward to define the parameters of the model when trying to predict real satellite conditions, moreover it assumes a constant speed distribution. Therefore, calculations of satellite drag using this model are restricted to one of the two limiting cases, and although intuitive, the validity of such an assumption is not clear [26]. Some practical applications of the Schamberg's model can be found in [35] and [21].

\subsubsection{Schaaf and Chambre derivation}

From Eqs. (18) and (19) we can obtain the reflected fluxes by means of the momentum accommodation coefficients as follows:

$$
\begin{gathered}
p_{r}=\left(1-\sigma_{N}\right) p_{i}+\sigma_{N} p_{w} \\
\tau_{r}=\left(1-\sigma_{T}\right) \tau_{i}
\end{gathered}
$$

Using the above relations together with Eqs. $(9-10)$ it is possible to derive a general expression for the pressure and shear stress coefficients for a flat plate 
with one side exposed to the flow. For the complete derivation, including the calculation of $p_{w}$ refer to [30]. The coefficients are given by (the reference area is the total area of the plate):

$$
\begin{gathered}
C_{p}=\frac{1}{s^{2}}\left\{\left(2-\sigma_{N}\right) \Gamma_{1}(s \cos \delta)+\frac{\sigma_{N}}{2}\left(\frac{T_{w}}{T_{\mathrm{inf}}}\right)^{1 / 2} \sqrt{\pi} \Gamma_{2}(s \cos \delta)\right\} \\
C_{\tau}=\frac{\sigma_{T} \sin \delta}{s} \Gamma_{2}(s \cos \delta)
\end{gathered}
$$

Where $T_{w}$ is the surface temperature and $T_{\mathrm{inf}}$ the ambient temperature, the $\Gamma$ functions are the ones defined in Eqs. $(11-12)$. Notice that there is no restriction on the angle of incidence $\delta$ therefore it may take values greater than 90 deg. In this scenario, the surface is aft facing. However, it may still be impacted by molecules that have large lateral thermal velocities in comparison to the bulk velocity vector.

Eqs. (29) and (30) depend on the two momentum accommodation coefficients $\left(\sigma_{N}, \sigma_{T}\right)$. They are meant to be determined by experiments, however, this approach is also far from being perfect: orbital conditions are hard to reproduce on earth facilities and orbital experiments are expensive and difficult to carry out. The reality is that little data have been gathered in representative conditions and a number of assumptions have to be made in order to give values to the coefficients. For a review of some experimental and theoretical approaches to determine these coefficients for different gases, surfaces and angles of incidence refer to $[12,13]$. Two practical cases, the determination of the CHAMP and ANS-1 aerodynamic databases, and the assumptions made to calculate the accommodation coefficients can be found in [26] and [18].

Alternative expressions for the force coefficients can be found in the literature. Storch [34] provides a very clear and easy to follow derivation of these equations, Sentman [33] assumes diffuse reflection and uses the temperature of the reflected molecules $\left(T_{r}\right)$ to account for incomplete thermal accommodation. Moe et al [24] modify Sentman's expressions to include the thermal accommodation coefficient instead of $T_{r}$. The different analytical formulations in the 
literature cannot always be reconciled. Care must be taken since it is critical to understand the basic assumptions under each of the formulas prior to producing any result. Some mistakes have been reported in the calculation of drag coefficients: confusion of kinetic and atmospheric temperatures in Cook's formula or incorrect hyperthermal assumption for certain flight conditions and body shapes [15].

Whereas the pressure coefficient has a unique definition, the drag and lift coefficients depend on the reference area chosen for the calculation. It is a common practice to use the projected area that varies with the incidence angle instead of a fixed area (however, the product $S C_{D}$ and hence the drag force are still the same regardless of the reference area chosen). A fixed reference area is preferred when building aerodynamic databases for dynamic simulations since it simplifies the calculation to retrieve the force. A projected reference area gives a better idea of the aerodynamic performance since it excludes the variation of projected area from $C_{D}$. It allows the comparison of $C_{D}$ for different vehicles or configurations.

An important comment is that different accommodation coefficients are associated with different gas-species, since different gas-surface interation is expected depending on the molecular characteristics of the gas. The atmosphere is a mixture of gases, and the drag coefficient is different for each constituent. Therefore, the drag force should be calculated taking into account a different drag coefficient for each species [12]. In practice, a different drag coefficient for each gas species introduces further uncertainties in the problem, a common approach being to use the mean molecular mass at the altitude of interest.

Besides the simple flat plate formula, it is also possible to derive analytical solutions of the force coefficients for some simple geometric shapes. The derivation consists on integrating the coefficients equations for an element of area over the surface of the body; see $[34,33,31]$. The analytical solutions are therefore limited to simple convex bodies such as spheres, cones, cylinders, etc. 


\subsection{Numerical Methods}

For more complex bodies, the aerodynamic coefficients have to be calculated by means of numeric methods. There are four main computational methods for analysing the aerodynamics of a body in free molecular flow:

- Panel Method

- Ray-Tracing Panel Method (RTP)

- Test-Particle Monte Carlo (TPMC)

- Direct Simulation Monte Carlo (DSMC)

They are compared in Fig. 14, along with the Computational Fluid Dynamics (CFD) method that is used in continuum flow. The right-hand axes of Fig. 14 represent both the free stream molecular speed ratio $s$ and free stream Knudsen number $K_{n}$. The Knudsen number is based on a spacecraft with a characteristic dimension of $5 \mathrm{~m}$. The different regimes of rarefied flow are indicated using the Knudsen number scale.

\subsubsection{Panel Method}

A body may be idealised as being made up of a number of discrete panels that can each be modelled as a flat plate with one side exposed to the flow. The pressure and shear stress for each panel can then be calculated using any of the available forms of the expressions for $C_{P}$ and $C_{\tau}$ for a flat plate in FMF. Then, adding the contributions of the $n$ panels all together it is possible to calculate the body force coefficient [29]:

$$
C_{F}=\sum_{i=1}^{n}\left\{C_{P_{i}}\left(-\overrightarrow{n_{i}}\right)+C_{\tau_{i}}\left[\overrightarrow{n_{i}} \times\left(\frac{\vec{V}}{|\vec{V}|} \times \overrightarrow{n_{i}}\right)\right]\right\} \frac{A_{i}}{A_{\text {ref }}}
$$

In hyperthermal flow only forward facing panels $(0 \leq \delta \leq \pi / 2)$ contribute to the body force coefficients. In contrast in hypothermal flow all panels may be impacted regardless of whether they are forward facing or not $(0 \leq \delta \leq \pi)$. This 


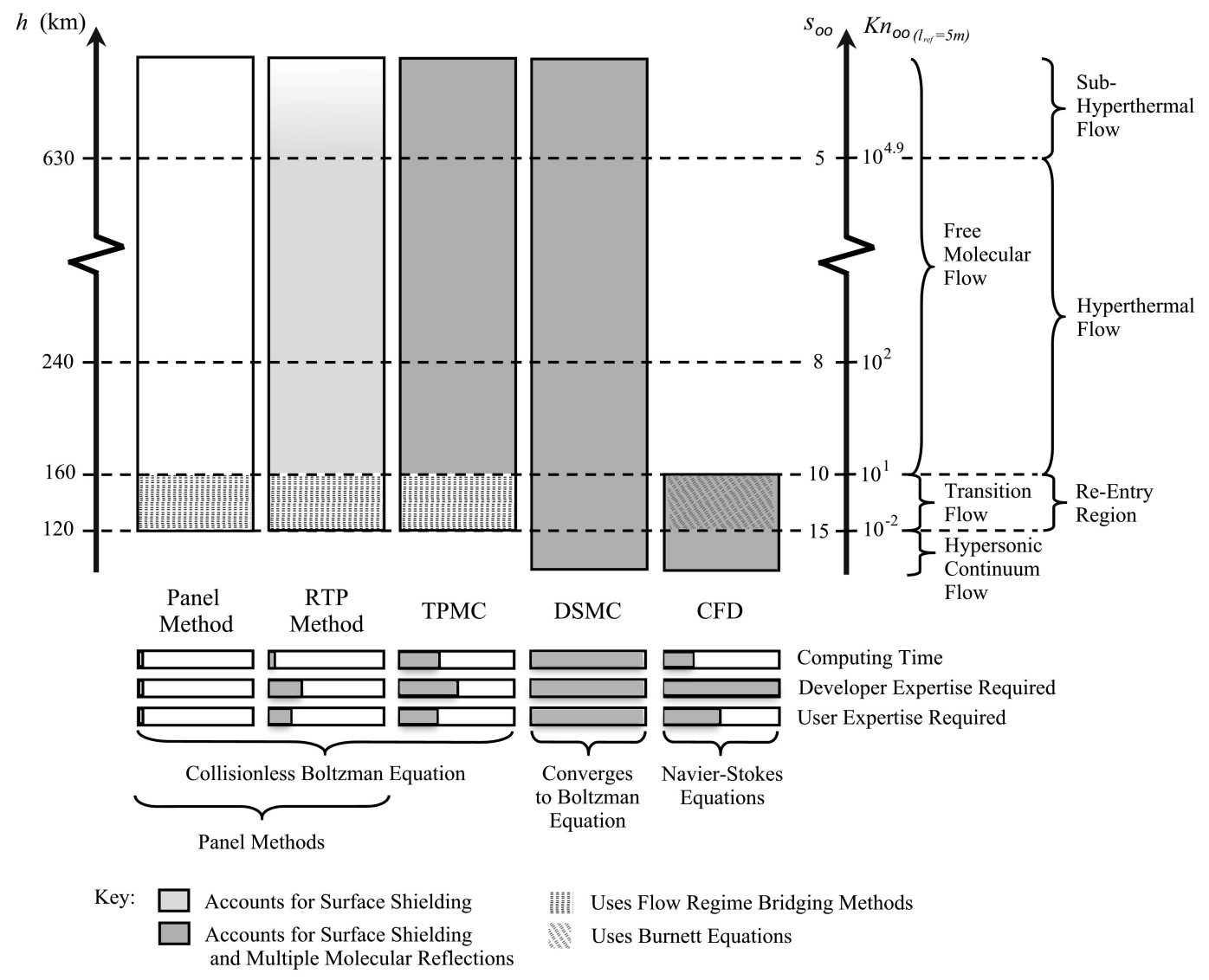

Figure 14: Comparison of existing computational approaches to spacecraft aerodynamics in low earth orbit 


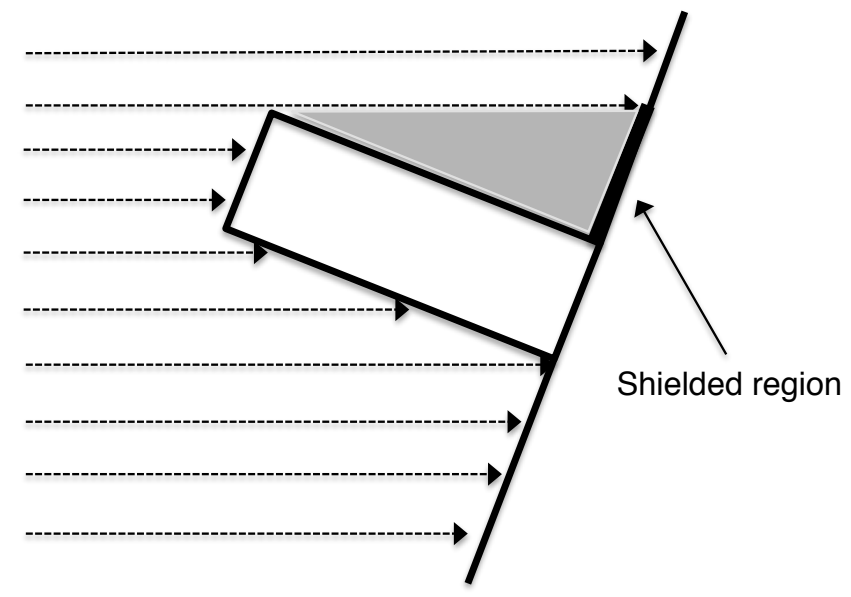

Figure 15: Surface Shielding

is because the molecules move with a velocity composed of their random thermal motion plus the bulk flow velocity. However, in practice, very few molecules will arrive at aft facing surfaces. Notice that it is possible to define more than one surface material in a certain mesh by defining groups of panels with different accommodation coefficients.

The panel method is only applicable for convex shapes. More complex shapes that have regions of concavity, or areas that are shielded to the flow (e.g as shown in Fig. 15) cannot be modelled realistically.

\subsubsection{RTP Method}

The Ray-Tracing Panel (RTP) method improves upon the panel method described in the previous section because it considers the shielding of spacecraft surfaces from the incident free stream flow by upstream components of the body. It can therefore be used to analyse more complex geometrical configurations.

The method assumes that the free stream flow behaves as a collimated beam of particles. This beam is analogous to a beam of light, such that areas shielded to the incident free stream flow (i.e. in shadow) can be determined using raytracing techniques. The next step is to remove these shadowed panels from the calculation and proceed with the non-shadowed ones in the same way as the 
panel method described above.

This method is only generally valid under hyperthermal conditions $(s>5)$. When modelling convex bodies with no shielded surfaces in hypothermal flow the random thermal motion of molecules is accounted for at the panel level in the GSIM. However, when modelling complex bodies with shielded surfaces, these are identified and removed from the calculation. Such a simplification assumption is no longer valid in hypothermal flow, so this method looses accuracy in this regime.

\subsubsection{TPMC Method}

The Test-Particle Monte Carlo (TPMC) is a step further with respect to panel methods. It was first proposed by Davis in 1961 [10]. In TPMC, the model is surrounded by a control tube into which a number of representative particles are sequentially fired. Each particle represents many thousands of real molecules. These test-particles, or simulated molecules, can be emitted from all sides of the control tube to mimic the characteristics of the real flow. Therefore, TPMC can be used to model the effects of multiple reflections and different flow conditions. The particles can reflect off the surfaces of the model, but do not interact with one another. Notice that this method needs a model of the scattering pattern of the reflected molecules (such as the ones developed by Maxwell or Schamberg).

The consequences of neglecting the effects of multiple reflections can be visualised with the help of Fig. 16; using the Panel or the RTP methods, the calculation of the force exerted on Surface A would yield the same result as the calculation of the force exerted on Surface B (assuming equivalent surface areas). Yet, in reality, the force exerted on Surface B would be less than the force exerted on Surface A because the surfaces of Surface A would be impacted by reflected flow in addition to free stream flow. 


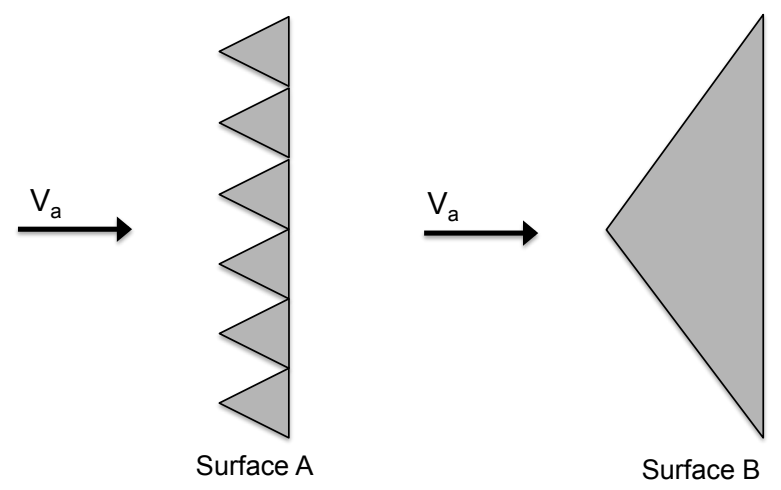

Figure 16: The importance of multiple reflections. If reflected molecules are ignored both surfaces will have the same force acting upon them. In reality, surface B will have less force exerted on it

\subsubsection{DSMC Method}

The Direct Simulation Monte Carlo (DSMC) was originally proposed by Graeme Bird [2]. The DSMC method directly simulates molecules. Each simulated molecule represents $10^{12}$ to $10^{20}$ real molecules. Unlike in TPMC, the simulated molecules can collide with one another. Collision samples and collision pairs are determined in a probabilistic manner. However, the movements of the molecules are deterministic and uncoupled from the collisions. Gas-surface interactions are handled using GSIMs and the body is typically represented by a surface mesh.

The simulation time period depends on the gas relaxation rate (the rate at which the gas returns to equilibrium conditions after a disturbance), and hence Knudsen number. Macroscopic quantities, such as temperature and pressure, can be calculated at each time step and then averaged to determine steady-state values. It has become the de facto method for modelling rarefied flow in the transition regime where the intermolecular collisions start to be of importance, such as re-entry flows. Excellent comparison between flight data and simulations results has been reported in many studies [7].

DSMC simulation results have also been proven to converge towards the 
Boltzmann equation [3]. Therefore, in theory, DSMC could be used to model flow in the continuum regime too. However, its computational load is directly proportional to the density of the gas. In the lower transition regime and continuum regime, this makes it prohibitively expensive in computational terms at the current time, especially for complex three dimensional bodies.

\section{Conclusion}

Although more than 60 years have passed since the first satellite was put in orbit, the drag force prediction of an orbiting spacecraft is still a challenging problem. There is still a requirement to improve our knowledge of the upper atmosphere environment and our understanding of the gas-surface interaction

phenomena. An increase on the interest in the topic is noticeable in recent years, both in the number of scientific missions with dedicated payloads and the number of published papers. Different methods of different complexity and accuracy have been reviewed in this paper to predict drag coefficients. Although they all depend on the accuracy of the gas-surface interaction model which is not always known, a comprehensive aerodynamic analysis of the spacecraft is of indubitable value to analyse and interpret gathered experimental data, as well as to study new ideas and proposals.

[1] Space engineering: Space environment, ECSS Std. ECSS-E-10-04A. ESAESTEC, Noordwijk, The Netherlands, 2008.

[2] G. A. Bird. Molecular gas dynamics and the direct simulation of gas flows, volume 42. Clarendon Press, Oxford, 1994.

[3] G. A. Bird. Forty years of DSMC, and now? In Rarefied Gas Dynamics: 22nd International Symposium, volume 585, page 372. American Institute of Physics, 9/7/2000 2000.

[4] B. R. Bowman. The semiannual thermospheric density variation from 1970 to 2002 between 200-1100 km. In Advances in the Astronautical Sciences, volume 119, pages 1135-1154, 2005. 
[5] B. R. Bowman, W. K. Tobiska, F. A. Marcos, C. Y. Huang, C. S. Lin, and W. J. Burke. A new empirical thermospheric density model JB2008 using new solar and geomagnetic indices. In AIAA/AAS Astrodynamics Specialist Conference and Exhibit, 2008.

[6] B. R. Bowman, W. Kent Tobiska, F. A. Marcos, and C. Valladares. The JB2006 empirical thermospheric density model. Journal of Atmospheric and Solar-Terrestrial Physics, 70(5):774-793, 2008.

[7] I. D. Boyd. Direct simulation Monte Carlo for atmospheric entry: Code development and application results. Technical Report EN-AVT-162, NATO RTO Report, 2009.

[8] G. E. Cook. Satellite drag coefficients. Planetary and Space Science, 13(10):929-946, 1965.

[9] R. Crowther and J. Stark. The determination of the gas-surface interaction from satellite orbit analysis as applied to ANS-1 (1975-70a). Planetary and Space Science, 39(5):729-736, 1991.

[10] D. H. Davis. Monte Carlo calculation of molecular flow rates through a cylindrical elbow and pipes of other shapes. Journal of Applied Physics, 31(7):1169-1176, 1960.

[11] E. Doornbos and H. Klinkrad. Modelling of space weather effects on satellite drag. Advances in Space Research, 37(6):1229-1239, 2006.

[12] E. M. Gaposchkin and Coster A.J. Analysis of satellite drag. Lincoln Laboratory Journal, 1:203-224, 1988.

[13] E. D. Knechtel and W. C. Pitts. Normal and tangential momentum accommodation for earth satellite conditions. Astronautica Acta, 18(3):171-184, 1973.

[14] S. H. Knowles, J. M. Picone, S. E. Thonnard, and A. C. Nicholas. The effect of atmospheric drag on satellite orbits during the Bastille Day Event. Solar Physics, 204(1-2):387-397, 2001. 
[15] G. Koppenwallner. Comment on special section: New perspectives on the satellite drag environments of Earth, Mars, and Venus. Journal of Spacecraft and Rockets, 45(6):1324-1327, 2008.

[16] F. A. Marcos, B. R. Bowman, and R. E. Sheehan. Accuracy of Earth's thermospheric neutral density models. In Collection of Technical Papers - AIAA/AAS Astrodynamics Specialist Conference, 2006, volume 1, pages 422-441, 2006.

[17] F. A. Marcos, W. J. Burke, and S. T. Lai. Thermospheric space weather modeling. In Collection of Technical Papers - 38th AIAA Plasmadynamics and Lasers Conference, volume 2, pages 999-1010, 2007.

[18] D. D. Mazanek, R. R. Kumar, M. Qu, and H. Seywald. Aerothermal analysis and design of the Gravity Recovery and Climate Experiment (GRACE) spacecraft. NASA Technical Memorandum, (210095):XI-42, 2000.

[19] D. D. Mazanek, R. R. Kumar, and H. Seywald. GRACE mission design: Impact of uncertainties in disturbance environment and satellite force models. In AAS/AIAA Space Flight Mechanics Meeting, volume AAS 00, page $163,20002000$.

[20] K. Moe. Six reasons why thermospheric measurements and models disagree. In M. H. Davis, Smith R.E., and Johnson D.L., editors, NASA Conference Publication, volume 2460, pages 291-303, feb 1987.

[21] K. Moe and B. R. Bowman. The effects of surface composition and treatment on drag coefficients of spherical satellites. In Advances in the Astronautical Sciences, volume 123 I, pages 137-152, 2006.

[22] K. Moe and M. M. Moe. Gas-surface interactions and satellite drag coefficients. Planetary and Space Science, 53(8):793-801, 2005.

[23] K. Moe and M. M. Moe. Gas-Surface Interactions in Low-Earth Orbit. In American Institute of Physics Conference Series, volume 1333 of American Institute of Physics Conference Series, pages 1313-1318, May 2011. 
[24] K. Moe, M. M. Moe, and C. J. Rice. Simultaneous analysis of multiinstrument satellite measurements of atmospheric density. Journal of Spacecraft and Rockets, 41(5):849-853, 2004.

[25] Oliver Montenbruck and Eberhard Gill. Satellite orbits: models, methods, and applications. Springer, Berlin, 2000. by Oliver Montenbruck and Eberhard Gill.; AERO.

[26] P. Moore and A. Sowter. Application of a satellite aerodynamics model based on normal and tangential momentum accommodation coefficients. Planetary and Space Science, 39(10):1405-1419, 1991.

[27] J. F. Padilla. Assessment of gas-surface interaction models for computation of rarefied hypersonic flows, 2008.

[28] J. M. Picone, A. E. Hedin, D. P. Drob, and A. C. Aikin. NRLMSISE-00 empirical model of the atmosphere: Statistical comparisons and scientific issues. Journal of Geophysical Research A: Space Physics, 107(A12), 2002.

[29] F. J. Regan and Satya M. Anandakrishnan. Dynamics of atmospheric reentry. American Institute of Aeronautics and Astronautics, Washington, DC, 2 edition, 1993. Frank J. Regan, Satya M. Anandakrishnan.; SPECBOOKS; ASE.

[30] S. A. Schaaf and P. L. Chambre. Flow of rarefied gases, volume 8. Princeton University Press, Princeton, NJ, 1961.

[31] S. A. Schaaf and L. Talbot. Mechanics of rarefied gases, volume 5 of Handbook of Supersonic Aerodynamics, chapter 16. Bureau of Naval Weapons U.S Navy, 1959.

[32] R. Schamberg. A new analytic representaticn of surface interaction with hypothermal free molecule flow with application to neutral-particle drag estimates of satellites. Technical Report RM-2313, RAND Research Memorandum, 1959. 
[33] L. H. Sentman. Free Molecule Flow Theory and Its Application to the Determination of Aerodynamic Forces. Lockheed Missiles and Space Company, Lockheed Aircraft Corporation, 1961.

[34] J. A. Storch. Aerodynamic disturbances on spacecraftin free-molecular flow (monograph). Technical report, The Aerospace Corporation, 2002.

[35] B. D. Tapley, J. C. Ries, S. Bettadpur, and M. Cheng. Neutral density measurements from the grace accelerometers. In Collection of Technical Papers - AIAA/AAS Astrodynamics Specialist Conference, 2006, volume 1, pages 484-494, 2006.

[36] K. F. Tapping. Recent solar radio astronomy at centimeter wavelengths: The temporal variability of the 10.7-cm flux. Journal of Geophysical Research, 92:829-838, January 1987.

[37] R. C. Tennyson. Atomic oxygen and its effect on materials in space. In International Symposium on Rarefied Gas Dynamics, volume 160, page 461, Vancouver, Canada, 31/7/1992 1992. AIAA, Rarefied Gas Dynamics: Space Science and Engineering.

[38] D. A. Vallado and D. Finkleman. A critical assessment of satellite drag and atmospheric density modeling. In AIAA/AAS Astrodynamics Specialist Conference and Exhibit, 2008.

[39] H. Y. Wachman. The thermal accommodation coefficient: A critical survey. Technical report, Space Sciences Laboratory, Missile and Space Vehicle Department, General Electric, 1961.

[40] Dean C. Wadsworth, Douglas B. Vangilder, and Virendra K. Dogra. Gassurface interaction model evaluation for DSMC applications. In Rarefied Gags Dynamics: 23rd International Symposium, volume 663, page 965. American Institute of Physics, 20/7/2002 2003.

[41] R. L. Walterscheid. Solar cycle effects on the upper atmosphere - implications for satellite drag. In AIAA, Aerospace Engineering Conference and 
Show, volume 26, pages 439-444. Journal of Spacecraft and Rockets, Feb. 14-16, 19891989.

[42] M. D. Zettergren, W. L. Oliver, P. L Blelly, and D. Alcayd. Modeling the behavior of hot oxygen ions. Annales Geophysicae, 24(6):1625-1637, 2006. 


\section{List of Figures}

1 Variation of the mean atmospheric density with altitude for low, moderate and high solar and geomagnetic activities as defined by JB2006 model. Reproduced from $[1] \ldots \ldots \ldots$

2 Variation of the Earth's atmospheric composition with altitude as defined by NRLMSISE00 model. Reproduced from [1] . . . . 6

3 Molecular Mean Free Path $(\lambda) \ldots \ldots \ldots$

4 Knudsen number variation with altitude for low, moderate and high solar and geomagnetic activities using NRLMSISE-00, and a characteristic dimension of $1 \mathrm{~m} \ldots \ldots \ldots$

5 Classification of flow regimes using Knudsen number. Reproduced from $[2] \ldots \ldots \ldots \ldots$

6 Hyperthermal $(s \rightarrow \infty)$ and hypothermal $(s<<\infty)$ flows $\ldots \ldots 11$

$7 \quad$ Impact on parallel surface due to random thermal motion of the flow molecules . . . . . . . . . . . . . . . . . . 11

8 Incident and reflected fluxes on a convex element of area . . . . 12

9 Specular and diffuse reflected fluxes $\ldots \ldots \ldots \ldots$

10 Schamberg's GSIM . . . . . . . . . . . . . . . . . 17

11 Schematic of the main environmental factors affecting accommodation coefficient and angular distribution in low earth orbit . . . 19

12 Uncertainties in drag coefficient caused by quasi-specular remission. Reproduced from $[22] \ldots \ldots$. . . . . . . . . 20

13 Schamberg's quasi-specular and quasi diffuse drag coefficients for simple geometries (based on projected area). Reproduced from [8] 22

14 Comparison of existing computational approaches to spacecraft aerodynamics in low earth orbit . . . . . . . . . . . . 26

15 Surface Shielding . . . . . . . . . . . . . . . . . 27

16 The importance of multiple reflections. If reflected molecules are ignored both surfaces will have the same force acting upon them. In reality, surface B will have less force exerted on it . . . . . . 29 